Article

\title{
Symmetry in Domination for Hypergraphs with Choice
}

\author{
Kenneth S. Berenhaut ${ }^{1}$ * , Brendan P. Lidral-Porter ${ }^{1}$, Theodore H. Schoen ${ }^{1}$ and Kyle P. Webb ${ }^{2}$ \\ 1 Department of Mathematics and Statistics, Wake Forest University, Winston-Salem, NC 27106, USA; \\ lidrbp15@wfu.edu (B.P.L.-P.); schot39@wfu.edu (T.H.S.) \\ 2 Department of Statistics, Virginia Tech University, Blacksburg, VA 24061, USA; webbkyle@vt.edu \\ * Correspondence: berenhks@wfu.edu; Tel.: +1-336-758-5922
}

Academic Editor: Angel Garrido

Received: 7 January 2017; Accepted: 14 March 2017; Published: 22 March 2017

\begin{abstract}
In this paper, we introduce the concept of (pair-wise) domination graphs for hypergraphs endowed with a choice function on edges. We are interested, for instance, in minimal numbers of edges for associated domination graphs. Theorems regarding the existence of balanced (zero-edge) domination graphs are presented. Several open questions are posed.
\end{abstract}

Keywords: hypergraph; choice; symmetry; hypertournament; domination

\section{Introduction}

In this paper, we introduce the concept of pair-wise domination for hypergraphs endowed with a choice function on edges. A hypergraph is a pair $\mathcal{H}=(V, E)$, where $V=\left\{v_{1}, v_{2}, \ldots, v_{n}\right\}$ is a set of $n$ vertices (or nodes) and $E=\left\{e_{1}, e_{2}, \ldots, e_{m}\right\}$ is a set of $m$ non-empty subsets of $V$ called hyperedges or edges (see for instance [1]). A $k$-hypertournament is a complete $k$-hypergraph $H=(V, E)$ (i.e., $E$ consists of all the $\left(\begin{array}{l}n \\ k\end{array}\right)$ possible $k$-subsets), with each $k$-edge endowed with an orientation. Here we are interested in hypergraphs where each edge, $e$, has a chosen element $C(e) \in e$ (in place of a complete orientation). We will refer to the pair $(H, C)$ as a (complete) $k$-hypergraph with choice (or an $(n, k)$ choice-hypergraph). For various considerations of choice functions, see for instance [2,3]. For some recent work related to choice in the context of Cayley graphs, see [4].

In the case $k=2$, both $k$-hypertournaments and hypergraphs with choice reduce to standard tournaments. For discussion of tournaments, see for instance [5-7]. The following is an example of a standard tournament with $n=5$ vertices.

Example 1. Consider the complete 2-hypertournament with five nodes and ten edges, depicted via the table in Figure 1a. Here, for instance, vertex 1 is chosen in the presence of vertices 2 and 4 (the first and third lines in the table). In fact, in this particular instance, each vertex is chosen for exactly two of the $\left(\begin{array}{l}5 \\ 2\end{array}\right)=10$ edges. Figure $1 b$ gives a graphical display with a directed edge from vertex $v$ to vertex $w$ whenever vertex $v$ is chosen in the presence of $w$.

In the next example, we consider a complete 3-hypergraph with choice.

Example 2. Consider the complete 3-hypergraph with choice with five vertices and 10 edges, depicted in the table in Figure 2a. Here, vertex 1 is chosen once in the presence of vertices 2 and 4 (for edges $\{1,2,3\}$ and $\{1,3,4\}$, respectively), twice in the presence of vertex 3 (again edges $\{1,2,3\}$ and $\{1,3,4\}$ ) and never in the presence of vertex 5. Note that again, as in Example 1, each node is chosen in the case of exactly two edges.

In considering possible analogues to Figure 1b, summarizing domination, one might include a directed edge from vertex $v$ to vertex $w$, if and only if, for edges that include both $v$ and $w$, the tally of wins for $v$ exceeds that 
of $w$. The resulting graph is depicted in Figure $2 b$. Note that there is a directed edge from vertex 1 to vertex 2 , since vertex 1 is chosen for edge $\{1,2,3\}$, while there is no edge, e, where vertex 2 is chosen when $1 \in e$. It may be noted that in this case the associated graph is path connected.

\begin{tabular}{|r||c||c||c|}
\hline & $\mathrm{e}$ & orientation & $\mathrm{C}(\mathrm{e})$ \\
\hline 1 & $\{1,2\}$ & $(1,2)$ & 1 \\
2 & $\{1,3\}$ & $(3,1)$ & 3 \\
3 & $\{1,4\}$ & $(1,4)$ & 1 \\
4 & $\{1,5\}$ & $(5,1)$ & 5 \\
5 & $\{2,3\}$ & $(2,3)$ & 2 \\
6 & $\{2,4\}$ & $(4,2)$ & 4 \\
7 & $\{2,5\}$ & $(2,5)$ & 2 \\
8 & $\{3,4\}$ & $(3,4)$ & 3 \\
9 & $\{3,5\}$ & $(5,3)$ & 5 \\
10 & $\{4,5\}$ & $(4,5)$ & 4 \\
\hline
\end{tabular}

(a)

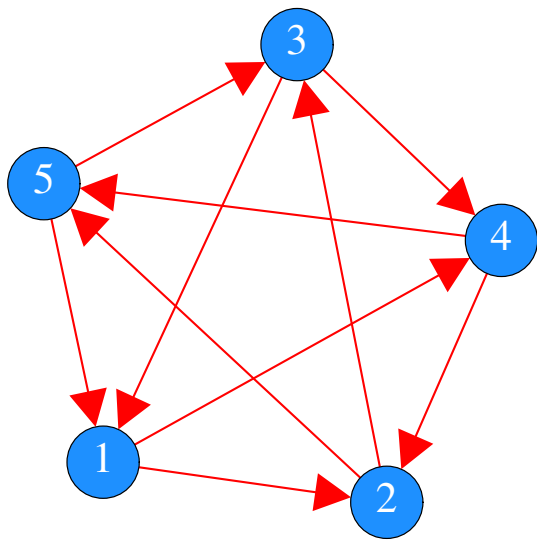

(b)

Figure 1. (a) An example of a standard tournament with five vertices; and (b) an associated graphical display with a directed edge from vertex $v$ to vertex $w$ whenever vertex $v$ is chosen in the presence of $w$.

\begin{tabular}{|r||c||c|}
\hline & $e$ & $C_{0}(e)$ \\
\hline 1 & $\{1,2,3\}$ & 1 \\
2 & $\{1,2,4\}$ & 4 \\
3 & $\{1,2,5\}$ & 5 \\
4 & $\{1,3,4\}$ & 1 \\
5 & $\{1,3,5\}$ & 3 \\
6 & $\{1,4,5\}$ & 4 \\
7 & $\{2,3,4\}$ & 3 \\
8 & $\{2,3,5\}$ & 2 \\
9 & $\{2,4,5\}$ & 2 \\
10 & $\{3,4,5\}$ & 5 \\
\hline
\end{tabular}

(a)

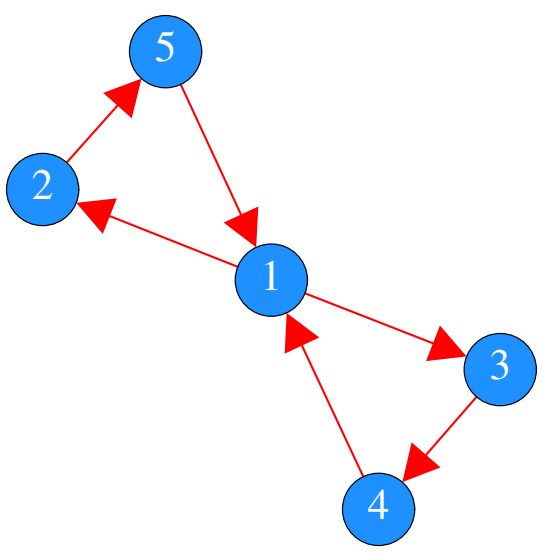

(b)

Figure 2. (a) An example of a possible $(5,3)$ choice-hypergraph; (b) with a possible graph summarizing pair-wise domination.

Now, define the function $\tau: V \times V \rightarrow \mathbb{Z}^{+}$via:

$$
\tau(v, w)=\mid\{e \in E: v, w \in e \text { and } C(e)=v\} \mid,
$$

i.e., $\tau(v, w)$ is the number of edges for which $v$ is chosen in the presence of $w$.

Example 2 leads to consideration of potential appropriate graphs on $n$ vertices reflecting domination properties among vertices. Here, we mention three possibilities.

(i) There is a directed edge from vertex $v$ to vertex $w$ if:

$$
\tau(w, v)=0
$$


i.e., if vertex $w$ is never chosen in the presence of vertex $v$.

(ii) There is a directed edge from vertex $v$ to vertex $w$ if:

$$
\tau(v, w)>\tau(w, v)
$$

i.e., among the edges containing both $v$ and $w, v$ is chosen with greater frequency.

(iii) There is a directed edge from vertex $v$ to vertex $w$ if:

$$
\tau(v, w)>\frac{\left(\begin{array}{c}
n-2 \\
k-2
\end{array}\right)}{2},
$$

i.e., $v$ is chosen for a majority of the edges containing both $v$ and $w$.

We will restrict attention henceforth to Option (ii), above, unless stated otherwise. It should be noted that for a standard tournament graph (i.e., $k=2$ ) all three formulations are equivalent; furthermore, Option (iii) is a stricter requirement than Option (ii). For discussion of ranking for vertices in hypertournaments, see for instance [8].

We refer to graphs as in Figures $2 \mathrm{~b}$ and 3 (below) as $(n, k)$-choice-domination graphs or simply $(n, k)$-domination graphs. When $n$ and $k$ are clear from context, we will at times simply refer to these as domination graphs. As with tournaments, domination graphs could be valuable in considerations of individual dominance in competitive settings, as may arise for instance in biology, game theory or decision analysis. Note that hypergraphs with choice allow for analysis of scenarios wherein selection (but not full orientation) information is available.

Example 3. Table 1 gives a 3-hypergraph with $n=5$ vertices and $m=10$ edges, along with four possible choice functions, $C_{0}$ (from Example 2), $C_{1}, C_{2}$ and $C_{3}$ on $E$, while Figure 3 includes the associated domination graphs, for comparison.

For fixed $n$ and $k$, many natural questions arise as to the properties of the resulting domination graphs; for instance:

1. What are the maximal and minimal number of edges possible for an $(n, k)$-domination graph?

2. What proportion of $(n, k)$-domination graphs are strongly path connected (for example, Figure $3 a, b$ ?

3. What is the distribution of the number of edges in the domination graph for a uniformly selected choice function on the edges of a $k$-hypergraph on $n$ vertices?

4. What is the number of non-isomorphic $(n, k)$-domination graphs?

Table 1. Four possible $(5,3)$ choice-hypergraphs.

\begin{tabular}{cccccc}
\hline & $e$ & $C_{0}(e)$ & $C_{1}(e)$ & $C_{2}(e)$ & $C_{3}(e)$ \\
\hline 1 & $\{1,2,3\}$ & 1 & 1 & 1 & 1 \\
2 & $\{1,2,4\}$ & 4 & 1 & 2 & 2 \\
3 & $\{1,2,5\}$ & 5 & 5 & 5 & 5 \\
4 & $\{1,3,4\}$ & 1 & 3 & 4 & 4 \\
5 & $\{1,3,5\}$ & 3 & 5 & 3 & 3 \\
6 & $\{1,4,5\}$ & 4 & 4 & 1 & 1 \\
7 & $\{2,3,4\}$ & 3 & 2 & 3 & 4 \\
8 & $\{2,3,5\}$ & 2 & 2 & 2 & 2 \\
9 & $\{2,4,5\}$ & 2 & 4 & 4 & 4 \\
10 & $\{3,4,5\}$ & 5 & 3 & 5 & 3 \\
\hline
\end{tabular}




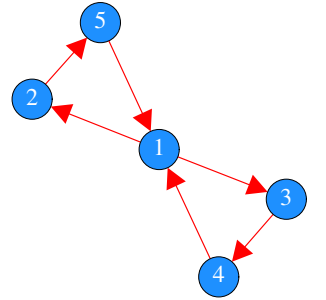

(a)

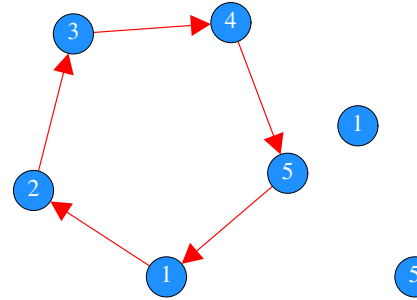

(b)

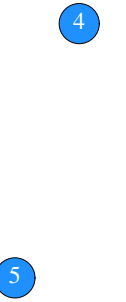

(c)

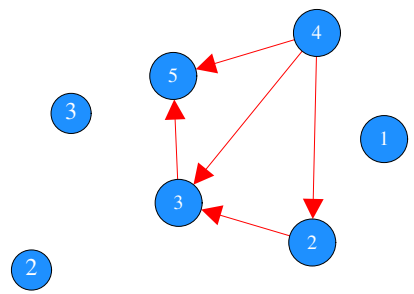

(d)

Figure 3. Domination graphs arising from choice-hypergraphs with choice functions (a) $C_{0}$; (b) $C_{1}$; (c) $C_{2}$; and (d) $C_{3}$ as in Table 1 .

Example 4. $((5,3)$ domination graphs.) For $(n, k)=(5,3)$, we have that $|E|=10$ and the number of distinct choice functions on $E$ is $3^{10}=59049$. Tables 2 and 3 give frequency tables for the number of choice functions leading to domination graphs with a given number of edges, and a given number of strongly connected components, respectively. Note that 3348 choice functions result in strongly connected domination graphs. There are 225 non-isomorphic (5,3)-domination graphs (of which 21 are strongly connected); plots of these are provided in the Supplementary Materials; Table 4 gives the frequencies for these graphs. The two most frequent domination graphs (each occurring for 1560 distinct choice functions, C), are given in Figure 4a,b respectively; the most frequently occurring strongly connected domination graph is given in Figure 4c.

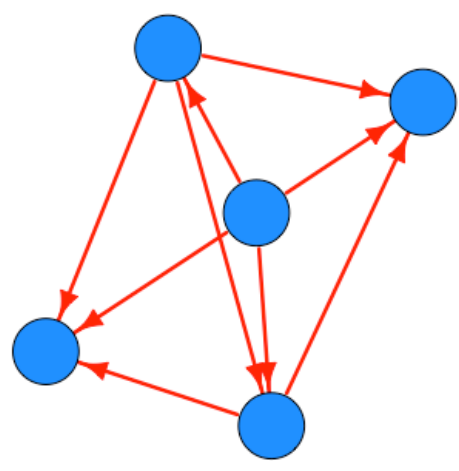

(a)

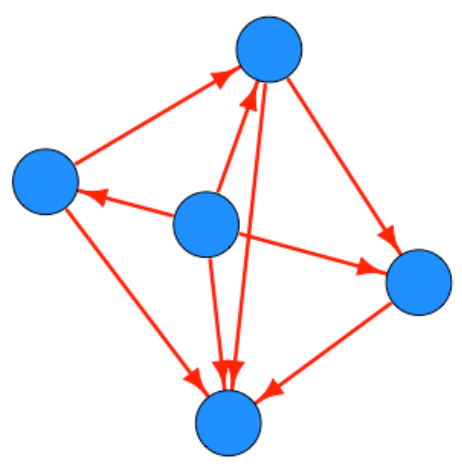

(b)

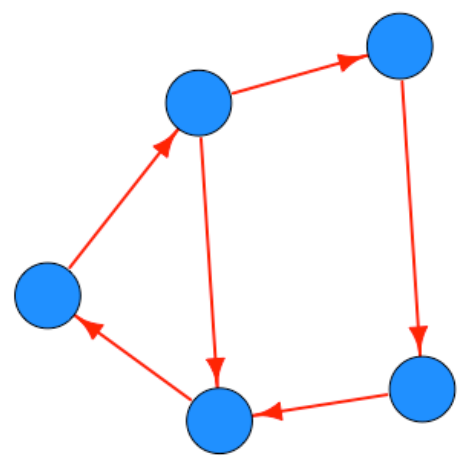

(c)

Figure 4. Three frequently occurring (5,3)-domination graphs. The two most frequent domination graphs are given in $(\mathbf{a}, \mathbf{b})$. The most frequently occurring strongly connected domination graph is given in (c).

Table 2. Edge distribution for (5,3)-domination graphs.

\begin{tabular}{ccccccccccc}
\hline Edges & 0 & 2 & 3 & 4 & 5 & 6 & 7 & 8 & 9 & 10 \\
\hline Frequency & 6 & 60 & 120 & 1035 & 3324 & 10080 & 15180 & 16920 & 9180 & 3144 \\
\hline
\end{tabular}

Table 3. Component distribution for (5,3)-domination graphs.

\begin{tabular}{ccccc}
\hline Components & 1 & 2 & 3 & 5 \\
\hline Frequency & 3348 & 6630 & 11760 & 37311 \\
\hline
\end{tabular}


Table 4. Frequency distribution for non-isomorphic (5,3)-domination graphs.

\begin{tabular}{|c|c|c|c|c|c|c|c|c|c|c|c|c|c|}
\hline Ind. & Freq. & Ind. & Freq. & Ind. & Freq. & Ind. & Freq. & Ind. & Freq. & Ind. & Freq. & Ind. & Freq. \\
\hline 1 & 1560 & 2 & 510 & 3 & 360 & 4 & 300 & 5 & 840 & 6 & 480 & 7 & 240 \\
\hline 8 & 480 & 9 & 120 & 10 & 600 & 11 & 840 & 12 & 240 & 13 & 960 & 14 & 120 \\
\hline 15 & 720 & 16 & 120 & 17 & 480 & 18 & 360 & 19 & 240 & 20 & 120 & 21 & 420 \\
\hline 22 & 840 & 23 & 240 & 24 & 120 & 25 & 120 & 26 & 960 & 27 & 180 & 28 & 180 \\
\hline 29 & 180 & 30 & 120 & 31 & 120 & 32 & 240 & 33 & 120 & 34 & 840 & 35 & 240 \\
\hline 36 & 480 & 37 & 240 & 38 & 120 & 39 & 600 & 40 & 120 & 41 & 240 & 42 & 360 \\
\hline 43 & 600 & 44 & 180 & 45 & 360 & 46 & 300 & 47 & 15 & 48 & 180 & 49 & 1200 \\
\hline 50 & 720 & 51 & 1560 & 52 & 240 & 53 & 960 & 54 & 480 & 55 & 120 & 56 & 360 \\
\hline 57 & 600 & 58 & 240 & 59 & 120 & 60 & 240 & 61 & 240 & 62 & 840 & 63 & 360 \\
\hline 64 & 840 & 65 & 480 & 66 & 840 & 67 & 360 & 68 & 120 & 69 & 240 & 70 & 720 \\
\hline 71 & 480 & 72 & 360 & 73 & 240 & 74 & 240 & 75 & 480 & 76 & 240 & 77 & 480 \\
\hline 78 & 360 & 79 & 120 & 80 & 240 & 81 & 120 & 82 & 240 & 83 & 120 & 84 & 120 \\
\hline 85 & 240 & 86 & 240 & 87 & 120 & 88 & 120 & 89 & 240 & 90 & 360 & 91 & 480 \\
\hline 92 & 360 & 93 & 480 & 94 & 120 & 95 & 240 & 96 & 240 & 97 & 120 & 98 & 120 \\
\hline 99 & 120 & 100 & 240 & 101 & 360 & 102 & 360 & 103 & 120 & 104 & 120 & 105 & 720 \\
\hline 106 & 240 & 107 & 120 & 108 & 210 & 109 & 120 & 110 & 240 & 111 & 120 & 112 & 120 \\
\hline 113 & 120 & 114 & 600 & 115 & 120 & 116 & 360 & 117 & 360 & 118 & 120 & 119 & 240 \\
\hline 120 & 360 & 121 & 120 & 122 & 120 & 123 & 120 & 124 & 120 & 125 & 240 & 126 & 120 \\
\hline 127 & 360 & 128 & 240 & 129 & 240 & 130 & 120 & 131 & 120 & 132 & 120 & 133 & 360 \\
\hline 134 & 120 & 135 & 240 & 136 & 300 & 137 & 120 & 138 & 120 & 139 & 120 & 140 & 60 \\
\hline 141 & 360 & 142 & 120 & 143 & 120 & 144 & 120 & 145 & 60 & 146 & 120 & 147 & 300 \\
\hline 148 & 240 & 149 & 120 & 150 & 120 & 151 & 360 & 152 & 240 & 153 & 240 & 154 & 120 \\
\hline 155 & 120 & 156 & 120 & 157 & 120 & 158 & 120 & 159 & 120 & 160 & 120 & 161 & 120 \\
\hline 162 & 120 & 163 & 240 & 164 & 120 & 165 & 480 & 166 & 120 & 167 & 240 & 168 & 240 \\
\hline 169 & 120 & 170 & 240 & 171 & 120 & 172 & 120 & 173 & 240 & 174 & 120 & 175 & 120 \\
\hline 176 & 120 & 177 & 240 & 178 & 120 & 179 & 120 & 180 & 120 & 181 & 240 & 182 & 120 \\
\hline 183 & 120 & 184 & 120 & 185 & 240 & 186 & 120 & 187 & 120 & 188 & 120 & 189 & 120 \\
\hline 190 & 120 & 191 & 120 & 192 & 120 & 193 & 120 & 194 & 120 & 195 & 120 & 196 & 120 \\
\hline 197 & 120 & 198 & 120 & 199 & 120 & 200 & 120 & 201 & 120 & 202 & 120 & 203 & 120 \\
\hline 204 & 120 & 205 & 120 & 206 & 120 & 207 & 120 & 208 & 120 & 209 & 120 & 210 & 60 \\
\hline 211 & 360 & 212 & 120 & 213 & 120 & 214 & 120 & 215 & 120 & 216 & 24 & 217 & 120 \\
\hline 218 & 120 & 219 & 120 & 220 & 120 & 221 & 120 & 222 & 240 & 223 & 120 & 224 & 24 \\
\hline 225 & 6 & & & & & & & & & & & & \\
\hline
\end{tabular}

In reference to Question 1 above, in Section 2 below, we will prove the following two results.

Theorem 1. Suppose $n, k \geq 1$ and $H=(V, E)$ is a complete $k$-hypergraph on $n$ vertices. If $k$ is odd and $\operatorname{gcd}(n, k)=1$, then there exists a choice function, $C$, on $E$ resulting in a zero-edge domination graph.

Theorem 2. If $(H, C)$ is a choice-hypergraph with a zero-edge domination graph, then for all $v \in V$ :

$$
|\{e \in E: C(e)=v\}|=\frac{1}{n} \cdot\left(\begin{array}{l}
n \\
k
\end{array}\right)
$$

that is, each vertex is chosen for an equal number of edges, in $E$.

The question of minimal edges in associated domination graphs may be of interest in instances where notions of "fairness" and equitable distribution are of importance, such as in resource allocation, decision theory, data and network processing, and clinical trials. Fairness and choice have been considered in the past, notably in the context of social welfare and information processing. The interested reader might like to consult, for instance [2-4,9-13].

Figure 5 provides an example of a $(9,5)$ choice-hypergraph with vertex set $V=\{1,2, \ldots, 9\}$, possessing a zero edge domination graph (employing the construction in the proof of Theorem 1 ). Note that $|E|=\left(\begin{array}{l}9 \\ 5\end{array}\right)=126$, and $\tau(1,2)=8=\tau(2,1)$ (as highlighted in red; $C(e)$ for $e \in E$ satisfying $\{1,2\} \in e$ are indicated in bold). It may also be verified that $|\{e \in E: C(e)=1\}|=126 / 9=14$, as required by Theorem 2 . 


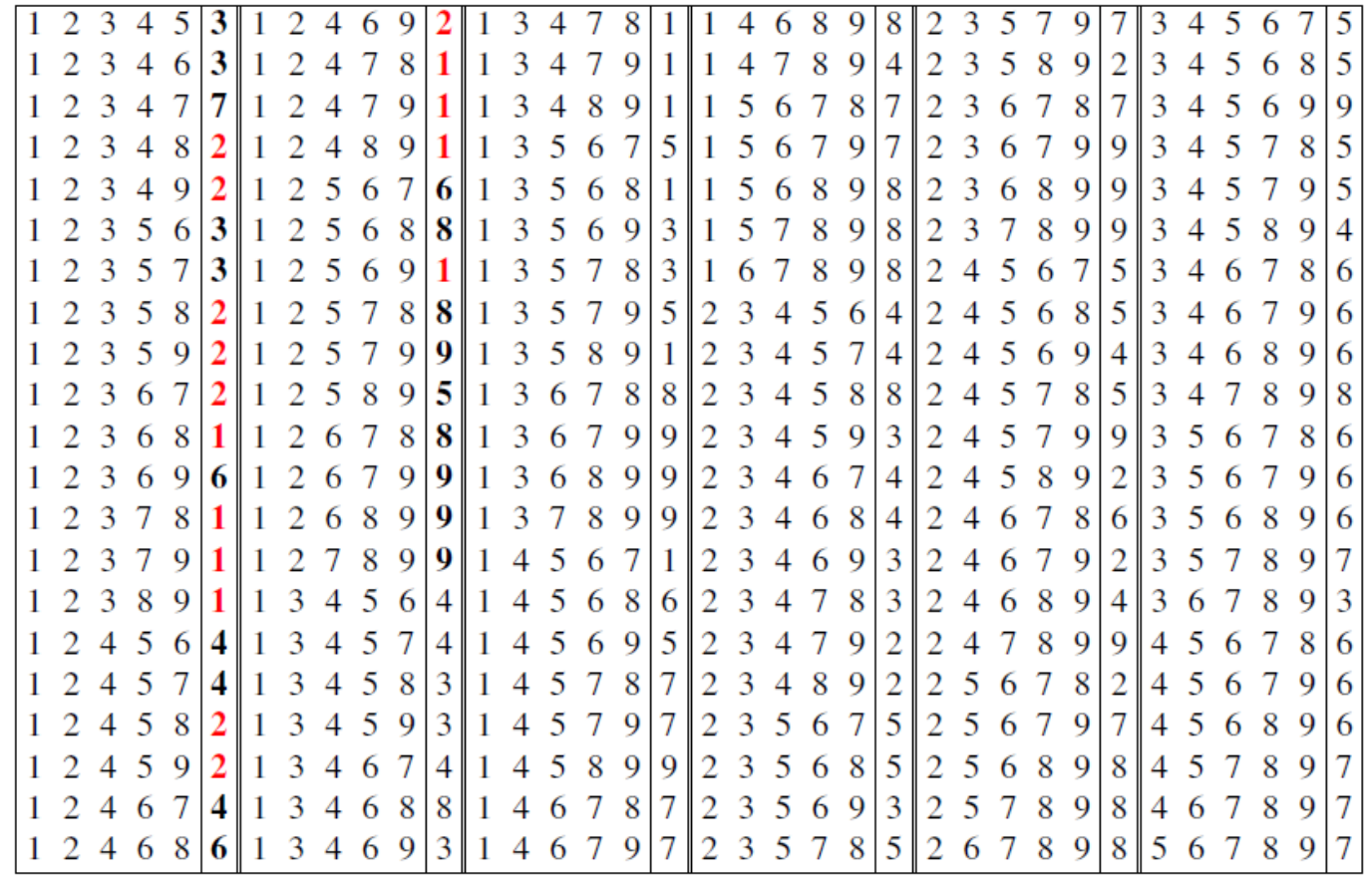

Figure 5. A (9,5)-choice hypergraph resulting in a zero-edge domination graph. For a given $e \in E$, the value of $C(e)$ is listed to the right of the five elements of $e$ in a demarcated column.

Before turning to the proofs of Theorems 1 and 2, we will briefly mention some recent related work on hypertournaments, which carry over to choice-hypergraphs. Recall that a $k$-hypertournament is a complete $k$-hypergraph $H=(V, E)$, with each edge endowed with an orientation. We will refer to the oriented edges as arcs.

One concept considered extensively in the literature is score sequences (see for instance $[6,7,14-18]$ ). In particular, for a given $1 \leq i \leq n$ define the score, $s_{i}$ of a vertex $v_{i}$ of a $k$-hypertournament on $H=(V, E)$ as the number of arcs containing $v_{i}$ in which $v_{i}$ is not the last element (this is with a complete orientation on the edges, rather than a choice function solely selecting a single element). Similarly, define the losing score, $r_{i}$ as the number of arcs containing $v_{i}$ in which $v_{i}$ is the last element. The total score, $t_{i}$, is then given by $t_{i}=s_{i}-r_{i}$. Finally, we obtain the score sequences $\left(s_{1}, \ldots, s_{n}\right)$, $\left(r_{1}, \ldots, r_{n}\right)$ and $\left(t_{1}, \ldots, t_{n}\right)$. Guofei et al. proved the following results regarding the existence of score sequences (see also $[16,19])$.

Theorem 3. (Guofei et al. [14]) Given two non-negative integers $n$ and $k$ with $n \geq k>1$, a non-decreasing sequence $R=\left(r_{1}, r_{2}, \ldots, r_{n}\right)$ of non-negative integers is a losing score sequence of some $k$-hypertournament if and only if for each $j(k \leq j \leq n)$ :

$$
\sum_{i=1}^{j} r_{i} \geq\left(\begin{array}{l}
j \\
k
\end{array}\right)
$$

with equality when $j=n$.

Theorem 4. (Guofei et al. [14]) Given two non-negative integers $n$ and $k$ with $n \geq k>1$, a non-decreasing sequence $S=\left(s_{1}, s_{2}, \ldots, s_{n}\right)$ of non-negative integers is a score-sequence of some $k$-hypertournament if and only if for each $j(k \leq j \leq n)$ :

$$
\sum_{i=1}^{j} s_{i} \geq j\left(\begin{array}{l}
n-1 \\
k-1
\end{array}\right)+\left(\begin{array}{c}
n-j \\
k
\end{array}\right)-\left(\begin{array}{l}
n \\
k
\end{array}\right)
$$


with equality when $j=n$.

A $k$-hypertournament is said to be regular if for each vertex, $v$, the tally of arcs containing $v$ as the last element is $\left(\begin{array}{l}n \\ k\end{array}\right) / n$. Koh and Ree [16] proved the following.

Theorem 5. (Koh and Ree, [16]) A regular $(n, k)$ hypertournament exists if and only if $n\left(\begin{array}{l}n \\ k\end{array}\right)$.

For alternative considerations of regularity, see $[8,20]$.

Compare Theorem 5 with Theorems 1 and 2, above. Note that symmetry in domination (i.e., the existence of choice functions resulting in zero-edge domination graphs) is a stronger requirement than regularity. To see this, simply note that all standard tournaments have $\left(\begin{array}{l}n \\ k\end{array}\right)$-edge domination graphs.

For further work on hypertournaments or score sequences, see for instance Pirizda et al. [15], Landau [18], Marshall [8], Khan et al. [6], Guofei et al. [14], Gunderson et al. [21], Li et al. [22], Guo and Surmacs [23], and Chou and Guofei [24].

In the next section, we prove Theorems 1 and 2.

\section{Proof of Theorems 1 and 2}

Before moving on to the proofs of Theorems 1 and 2, we introduce some preliminary notation. First, suppose $n \geq k \geq 1$ are fixed and $(H, C)$ is an $(n, k)$-hypergraph with choice, where $H=(V, E)$ is a complete $k$-hypergraph on $n$ vertices. Without loss of generality, we assume that $V=\{0,1, \ldots, n-1\}$. Similar to in [16], define the rotation operator $P: E \rightarrow E$, via $P(e)=e+1(\bmod n)$, i.e., $P$ acts on $k$-subsets of $V$ by shifting the elements (cyclically) to the right by one. Here, $e+1$ indicates $\{v+1: v \in e\}$.g For $e \in E$ and $j \geq 0$, define $P^{j}$ as the $j$-fold iteration of $P$ and the order of $e, \alpha_{e}$, via:

$$
\alpha_{e} \stackrel{\text { def }}{=} \min \left\{\gamma \geq 1: P^{\gamma}(e)=e\right\}
$$

We will denote the set of equivalence classes under successive application of $P$ by $\mathcal{R}=$ $\left\{R_{1}, \ldots, R_{q}\right\}$. Note that for $R \in \mathcal{R},|R|$ is the order of each $e \in R$. We will refer to elements of $\mathcal{R}$ as rotation classes of $H$.

In general, addition of the form $S+c$ for $S \subseteq \mathbb{Z}$ and $c \in \mathbb{Z}$, will be modulo $n$, unless stated otherwise.

The set $e \in E$ is said to be symmetric if $-e=e(\bmod n)$, and more generally $R \in \mathcal{R}$ is symmetric if, for all $e \in R,-e \in R$. Note that if for some $e \in R, e=-e$, then for $1 \leq i \leq n,-(e+i)=-e-i=$ $e-i \in R$, and hence if $e \in R$ is symmetric, then $R$ is symmetric. If $R$ is not symmetric, then there exists an $R^{\prime} \in \mathcal{R} \backslash R$ such that $e \in R$ implies $-e \in R^{\prime}$.

Let $\mathcal{H}_{n, k}$ be the set of all $(n, k)$-choice hypergraphs for fixed $n, k \in \mathbb{Z}^{+}$and $\mathcal{G}_{n}$ be the set of all directed graphs on $n$ vertices. Suppose a domination scheme, $D$ is fixed (see (i)-(iii), above, for examples) and define $G_{D}: \mathcal{H}_{n, k} \rightarrow \mathcal{G}_{n}$, where $G_{D}(T)$ is the domination graph for choice-hypergraph $T=(H, C)$ under domination scheme $D$.

We have the following elementary lemma:

Lemma 1. Suppose $H$ is a $k$-hypertournament on $n$ vertices with $\operatorname{gcd}(n, k)=1$. Then, for any $R \in \mathcal{R}$, $|R|=n$.

Proof. Suppose $\operatorname{gcd}(n, k)=1$, and $\mathcal{R}=\left\{R_{1}, R_{2}, \ldots, R_{q}\right\}$ for some $q \geq 1$. For any $R \in \mathcal{R}$ and any $e \in R$, set $\alpha=\alpha_{e}$ and represent $e$ as a binary vector $\zeta=\left(\zeta_{1}, \zeta_{2}, \ldots, \zeta_{n}\right)$. We then have, with $a=\Sigma_{1 \leq i \leq \alpha} \zeta_{i}$ and $b=n / \alpha$ :

$$
k=b a, \quad n=b \alpha .
$$

Now, suppose $n=\alpha p+r$, with $p \geq 0$ and $0 \leq r \leq \alpha-1$. Then:

$$
e=P^{\alpha(p+1)}(e)=P^{n-r+\alpha}(e)=P^{\alpha-r}(e) .
$$


Since $\alpha$ is minimal, we have $r=0$ and hence $b=n / \alpha \in \mathbb{Z}$. Thus, since $g c d(n, k)=1$, (9) gives that $b=1, \alpha=n$ and finally $|R|=n$.

For convenience of notation, as in (1), define the function $\tau_{R}: V \times V \rightarrow \mathbb{Z}^{+}$via:

$$
\tau_{R}(v, w)=\mid\{e \in R: v, w \in e \text { and } C(e)=v\} \mid,
$$

i.e., $\tau_{R}(v, w)$ is the number of edges in the rotation class $R \in \mathcal{R}$ for which $v$ is chosen in the presence of $w$. Note that

$$
\tau(v, w)=\sum_{R \in \mathcal{R}} \tau_{R}(v, w)
$$

Lemma 2. Suppose $n, k \geq 1$ with $k$ odd, $H=(V, E)$ is a complete $k$-hypergraph on $n$ vertices, and $R \in \mathcal{R}$. If $R$ is symmetric and $e \in R$, then there exists $a \xi \in V$ and $\mathcal{T}=\left\{\delta_{1}, \delta_{2}, \ldots, \delta_{k}\right\}$, such that $e=\xi+\mathcal{T}$, and $\delta \in \mathcal{T}$ implies $-\delta \in \mathcal{T}$.

Proof. Suppose that $R$ is symmetric and $e=\left\{x_{1}, \ldots, x_{k}\right\} \in R$. Then, there exists an $i$ such that $e+i=-e$, and hence a permutation $\left(j_{1}, j_{2}, \ldots, j_{k}\right)$ of $(1,2, \ldots, k)$ such that:

$$
x_{j_{q}}+i=-x_{q}, \quad 1 \leq q \leq k .
$$

Since $k$ is odd, there exists a $Q$ such that:

$$
x_{Q}+i=-x_{Q}
$$

and taking differences, (13) and (14) imply:

$$
x_{j_{q}}-x_{Q}=x_{Q}-x_{q}=-\left(x_{q}-x_{Q}\right), \quad 1 \leq q \leq k .
$$

The result follows upon setting $\xi=x_{Q}$, and $\mathcal{T}=\left\{x_{q}-x_{Q}: 1 \leq q \leq k\right\}$.

We will now prove Theorem 1 regarding the existence of choice functions with symmetry in domination.

Proof of Theorem 1. Suppose $\operatorname{gcd}(n, k)=1, k$ is odd, and $(H, C)$ is an $(n, k)$-choice hypergraph. Consider $E$, the set of all edges in $H$, and let $\mathcal{R}=\left\{R_{1}, R_{2}, \ldots, R_{q}\right\}$ be the set of all rotation classes of $H$, where, by Lemma 1 , for $R \in \mathcal{R},|R|=n$, and $|\mathcal{R}|=\left(\begin{array}{l}n \\ k\end{array}\right) / n$. Fix $v, w \in V$, with $v \neq w$, and choose some $R \in \mathcal{R}$.

Suppose $R$ is symmetric and fix an $e \in R$. Then, by Lemma 2, $e=\xi+\mathcal{T}$, where $\xi \in V$ and $\mathcal{T}$ is closed under additive inverses. For $u=e+i \in R$, set $C(u)=\xi+i$. Suppose $v=\xi+j_{v}$ and $w=\xi+j_{w}$ for $j_{v}, j_{w} \in\{0,1, \ldots, n-1\}$. For $f \in R, C(f)=v$ implies $f=e+j_{v}$ and $C(f)=w$ implies $f=e+j_{w}$. Note that $w \in e+j_{v}$ if and only if $j_{w}-j_{v} \in \mathcal{T}$. Similarly, $v \in e+j_{w}$ if and only if $j_{v}-j_{w} \in \mathcal{T}$. Since $\mathcal{T}$ is closed under inverses, we have:

$$
\begin{aligned}
\tau_{R}(v, w) & =\mid\{e \in R: v, w \in e \text { and } C(e)=v\} \mid \\
& =\mid\{e \in R: v, w \in e \text { and } C(e)=w\} \mid \\
& =\tau_{R}(w, v) .
\end{aligned}
$$

Suppose $R$ is not symmetric, and consider $R^{\prime}=\{-e: e \in R\}$, and note that $R \cap R^{\prime}=\varnothing$. Fix an $e \in R$ and $\xi \in e$, and write $e=\xi+\mathcal{T}$ (note that $\mathcal{T}$ is not closed under inverses). For $f \in R \cup R^{\prime}$, set: 


$$
C(f)= \begin{cases}\xi+i & \text { if } f=e+i=\xi+i+\mathcal{T} \in R \\ -\xi+i & \text { if } f=-e+i=-\xi+i-\mathcal{T} \in R^{\prime} .\end{cases}
$$

Now, suppose $v=\xi+j_{v}$ and $w=\xi+j_{w}$. For $f \in R \cup R^{\prime}, C(f)=v$ implies $f=e+j_{v}$ or $f=-e+j_{v}$, and $C(f)=w$ implies $f=e+j_{w}$ or $f=-e+j_{w}$. Note that $w \in e+j_{v}$ if and only if $j_{w}-j_{v} \in \mathcal{T}$, and $w \in-e+j_{v}$ if and only if $j_{w}-j_{v} \in-\mathcal{T}$ (i.e., $j_{v}-j_{w} \in \mathcal{T}$ ). Similarly, $v \in e+j_{w}$ if and only if $j_{v}-j_{w} \in \mathcal{T}$, and $v \in-e+j_{w}$ if and only if $j_{v}-j_{w} \in-\mathcal{T}$ (i.e., $j_{w}-j_{v} \in-\mathcal{T}$ ). Thus:

$$
\begin{aligned}
\tau_{R \cup R^{\prime}}(v, w) & =\mid\left\{f \in R \cup R^{\prime}: v, w \in f \text { and } C(f)=v\right\} \mid \\
& =\mid\left\{f \in R \cup R^{\prime}: v, w \in f \text { and } C(f)=w\right\} \mid \\
& =\tau_{R \cup R^{\prime}}(w, v) .
\end{aligned}
$$

Employing (16), (18) and (12), the result follows.

We will now prove Theorem 2.

Proof of Theorem 2. Suppose $(H, C)$ is a choice-hypergraph with a zero-edge domination graph, where $H=(V, E)$ is a complete $k$-hypergraph on $n$ vertices. For a fixed vertex $v \in V$, define $\sigma_{v}=\{e \in E: v \in e\}$ and $\omega_{v}=\left\{e \in \sigma_{v}: C(e)=v\right\}$, i.e., $\sigma_{v}$ is the set of edges to which $v$ belongs and $\omega_{v}$ is the set of edges for which $v$ is selected. Note that:

$$
\left|\omega_{v}\right| \leq\left|\sigma_{v}\right|=\left(\begin{array}{l}
n-1 \\
k-1
\end{array}\right)
$$

Assume $\left|\omega_{v}\right|<\left(\begin{array}{l}n \\ k\end{array}\right) / n$. Since $(H, C)$ has a zero-edge domination graph, $\tau(w, v)=\tau(v, w)$ for all $w \in V$ and hence:

$$
\begin{aligned}
\left(\begin{array}{l}
n-1 \\
k-1
\end{array}\right) & =\left|\sigma_{v}\right|=(k-1)\left|\omega_{v}\right|+\left|\omega_{v}\right| \\
& <k \frac{1}{n}\left(\begin{array}{l}
n \\
k
\end{array}\right)=\left(\begin{array}{l}
n-1 \\
k-1
\end{array}\right) .
\end{aligned}
$$

Thus, $\left|\omega_{v}\right| \geq\left(\begin{array}{l}n \\ k\end{array}\right) / n$ for all $v \in V$. The result follows upon noting that:

$$
\sum_{v \in V} \omega_{v}=|E|=\left(\begin{array}{l}
n \\
k
\end{array}\right)
$$

Supplementary Materials: The following are available online at http:/ /www.mdpi.com/2073-8994/9/3/46/s1, File S1: Plots of the 225 distinct non-isomorphic $(5,3)$ domination graphs. File S2: Plots of the 21 distinct strongly connected, non-isomorphic $(5,3)$ domination graphs

Author Contributions: Kenneth S. Berenhaut, Brendan P. Lidral-Porter, Theodore H. Schoen and Kyle P. Webb contributed to the results; Kenneth S. Berenhaut and Brendan P. Lidral-Porter wrote the paper.

Conflicts of Interest: The authors declare no conflict of interest.

\section{References}

1. Berge, C.; Minieka, E. Graphs and Hypergraphs; North-Holland Publishing Company: Amsterdam, The Netherlands, 1973; Volume 7.

2. Johnson, M.R. Ideal structures of path independent choice functions. J. Econ. Theory 1995, 65, 468-504.

3. Moulin, H. Choice functions over a finite set: A summary. Soc. Choice Welf. 1985, 2, 147-160.

4. Beeler, K.E.; Berenhaut, K.S.; Cooper, J.N.; Hunter, M.N.; Barr, P.S. Deterministic walks with choice. Discret. Appl. Math. 2014, 162, 100-107. 
5. Kayibi, K.; Khan, M.A.; Pirzada, S. Uniform sampling of k-hypertournaments. Linear Multilinear Algebra 2013, 61, 123-138.

6. Khan, M.A.; Pirzada, S.; Kayibi, K.K. Scores, inequalities and regular hypertournaments. J. Math. Inequal. Appl. 2012, doi: 10.7153/mia-15-28.

7. Pirzada, S.; Naikoo, T. On score sets in tournaments. Vietnam J. Math. 2006, 34, 157-161.

8. Marshall, S. Properties of K-Tournaments. Ph.D. Thesis, Simon Fraser University, Burnaby, BC, Canada, 1994.

9. Brookes, S. Fairness, resources, and separation. Electron. Notes Theor. Comput. Sci. 2010, 265, 177-195.

10. Cooper, C.; Ilcinkas, D.; Klasing, R.; Kosowski, A. Derandomizing random walks in undirected graphs using locally fair exploration strategies. Distrib. Comput. 2011, 24, doi: 10.1007/s00446-011-0138-4.

11. Jaeger, M. On fairness and randomness. Inf. Comput. 2009, 207, 909-922.

12. Krawczyk, M.W. A model of procedural and distributive fairness. Theory Decis. 2011, 70, 111-128.

13. Völzer, H.; Varacca, D. Defining fairness in reactive and concurrent systems. J. ACM 2012, 59, doi: 10.1145/2220357.2220360.

14. Guofei, Z.; Tianxing, Y.; Kemin, Z. On score sequences of k-hypertournaments. Eur. J. Comb. 2000, 21, 993-1000.

15. Pirzada, S.; Chishti, T.; Naikoo, T. Score lists in [h-k]-bipartite hypertournaments. Discret. Math. Appl. 2009, 19, 321-328.

16. Koh, Y.; Ree, S. On k-hypertournament matrices. Linear Algebra Appl. 2003, 373, 183-195.

17. Pirzada, S.; Khan, M.A.; Guofei, Z.; Kayibi, K.K. On scores, losing scores and total scores in hypertournaments. Electron. J. Graph Theory Appl. 2015, 3, 8-21.

18. Landau, H. On dominance relations and the structure of animal societies: III The condition for a score structure. Bull. Math. Biol. 1953, 15, 143-148.

19. Pirzada, S.; Zhou, G. On k-hypertournament losing scores. Acta Univ. Sapientiae 2010, 2, 5-9.

20. Surmacs, M. Regular hypertournaments and Arc-pancyclicity. J. Graph Theory 2017, 84, 176-190.

21. Gunderson, K.; Morrison, N.; Semeraro, J. Bounding the number of hyperedges in friendship r-hypergraphs. Eur. J. Comb. 2016, 51, 125-134.

22. Li, H.; Li, S.; Guo, Y.; Surmacs, M. On the vertex-pancyclicity of hypertournaments. Discret. Appl. Math. 2013, 161, 2749-2752.

23. Guo, Y.; Surmacs, M. Pancyclic out-arcs of a vertex in a hypertournament. Australas. J. Comb. 2015, 61, 227-250.

24. Chao, W.; Guofei, Z. Note on the degree sequences of k-hypertournaments. Discret. Math. 2008, 308, 2292-2296.

(C) 2017 by the authors. Licensee MDPI, Basel, Switzerland. This article is an open access article distributed under the terms and conditions of the Creative Commons Attribution (CC BY) license (http:/ / creativecommons.org/licenses/by/4.0/). 